\title{
PRESCRIPCIÓN INADECUADA DE TRANSFUSIÓN SANGUÍNEA EN UN HOSPITAL DE REFERENCIA DE LIMA, PERÚ
}

\author{
Wilfredo Flores-Paredes $1, a$
}

\begin{abstract}
RESUMEN
Objetivos. Determinar la frecuencia y los criterios clínicos asociados a la prescripción inadecuada de hemocomponentes. Materiales y métodos. Estudio transversal realizado entre junio y octubre de 2002. Se revisó las historias médicas y los exámenes de laboratorio de los pacientes que recibieron transfusiones sanguíneas. Para valorar las indicaciones de transfusión como adecuadas o inadecuadas se empleó una guía de transfusión elaborada en base a consensos publicados. Resultados. Se evaluaron 311 indicaciones de transfusión. El porcentaje global de prescripción inadecuada fue de $33.8 \%$. Los glóbulos rojos (GR) se usaron inadecuadamente en $25 \%$, el plasma fresco congelado en $59 \%$, las plaquetas en $13 \%$ y el crioprecipitado en $88 \%$. En Cirugía, una de cada dos indicaciones fue inadecuada mientras que en los servicios de Medicina, $\mathrm{UCl}$ y Emergencia, una de cada tres lo fue. El plasma fresco congelado fue el componente peor utilizado. Conclusiones. Existe una elevada frecuencia de prescripción inadecuada de hemocomponentes en la población de estudio. La identificación de los criterios clínicos específicos de indicación inapropiada de transfusión puede ayudar a diseñar estrategias para el uso racional.
\end{abstract}

Palabras clave: Transfusión de componentes sanguíneos; Seguro social; Guías de práctica médica (fuente: DeCS BIREME).

\section{TO INADEQUATE INDICATION OF BLOOD TRANSFUSION IN A REFERENCE HOSPITAL IN LIMA, PERU}

\begin{abstract}
Objectives. To determine frequency and clinical criteria associated to the inadequate indication of blood products. Materials and methods. A cross-sectional study was performed between June and October 2002. Clinical charts and the laboratory tests from patients receiving blood transfusions were examined. A blood transfusion guideline was elaborated based on published consensus, and used to determine whether the indications for blood products transfusion was adequate or inadequate. Results. A total of 311 blood transfusions were evaluated. The global prevalence of inappropriate indication was of $33.8 \%$. Red blood cells (RBC) were used inappropriately in $25 \%$, fresh frozen plasma (FFP) in $59 \%$, platelets in $13 \%$ and cryoprecipitate in $88 \%$. From surgery related transfusions half of the indications were inadequate. In internal medicine, ICU and emergency related transfusions, approximately one third of the indications were inadequate. The FFP is the worst used blood product. Conclusions. We found a high frequency of inadequate blood transfusion. The identification of the specific clinical criteria used in inadequate indications transfusions will help in designing a better educational strategy towards a more rational use of the blood products.
\end{abstract}

Key words: Blood component transfusion; Social security; Practice guidelines (source: MeSH NLM).

\section{INTRODUCCIÓN}

Los beneficios de la transfusión sanguínea son reales y de su uso puede depender la vida del paciente; sin embargo, no está libre de riesgos, aunque ha adquirido un gran desarrollo y seguridad, aún no es posible proporcionar un componente sanguíneo con riesgo cero (1). Entre los efectos adversos más conocidos de la transfusión sanguínea se encuentran las reacciones febriles no hemolíticas, hemólisis inmune, aloinmunización, enfermedad de injerto contra huésped, inmunomodulación e infecciones. En el uso de los hemocomponentes es necesario un análisis riesgo/ beneficio para el paciente, por lo que cada indicación requiere una valoración profunda que garantice un mejor aprovechamiento y éxito de la hemoterapia (2).

Un problema presente en la práctica de la transfusión corriente es la elevada proporción de transfusiones que son catalogadas como innecesarias. En los Estados Unidos de Norteamérica se informan tasas de $57 \%$ en transfusión inapropiadas de glóbulos, de $71 \%$ en plasma fresco congelado y hasta el $26 \%$ en concentrados plaquetarios ${ }^{(3-5)}$. Metz et al. ${ }^{(6)}$, en una auditoría realizada a un hospital de Australia, identificaron que las tasas inapropiadas de transfusión de GR fueron

1 Departamento de Patología Clínica, Hospital Nacional Guillermo Almenara Irigoyen, EsSalud. Lima, Perú.

a Médico Patólogo Clínico, Magíster en Epidemiología 
de $16 \%$, de plaquetas en $13 \%$, y de PFC en $24 \%$. En Ontario Canadá ${ }^{(7)}$, se halló que $55,3 \%$ de las unidades de GR tuvieron un uso innecesario. Otro estudio en Canadá ${ }^{(8)}$, halló que el $45 \%$ de las transfusiones de PFC fueron inapropiadas. Mientras que en Francia ${ }^{(9)}$ una auditoría juzgó que el $23 \%$ de las prescripciones de PFC fueron inapropiadas.

Durante el año 2001 fueron transfundidos más de 65000 componentes sanguíneos en el Hospital Nacional Edgardo Rebagliati Martins, hospital de referencia del seguro social de Perú. El objetivo de la presente investigación fue conocer la frecuencia de inadecuada prescripción de los componentes sanguíneos así como conocer los criterios clínicos específicos de estas indicaciones innecesarias.

\section{MATERIALES Y MÉTODOS}

Estudio transversal descriptivo, realizado en el Hospital Nacional Edgardo Rebagliati Martins, Lima, Perú. Este hospital cuenta con 1492 camas de hospitalización y con casi todas las especialidades médicas y quirúrgicas. La población de estudio lo conformaron pacientes mayores de 14 años que recibieron alguna transfusión sanguínea durante los meses de junio a octubre de 2002. Se excluyeron a los pacientes con anemia hemolítica congénita (talasemias) o adquirida, que fueron transfundidos con glóbulos rojos, ya que requieren criterios de indicación muy específicos.

En la presente investigación se consideraron cuatro servicios: Medicina (Med), Unidad de Cuidados Intensivos (UCl), Cirugía Hospitalización (Cir-Hosp), Cirugía Sala de Operaciones (Cir-SOP) y Emergencia (Emg). En el periodo entre junio a octubre de 2002, se prescribieron aproximadamente 9000 unidades por 3000 indicaciones de transfusión. Considerando que la proporción esperada de transfusión inadecuada era del $50 \%$, con una precisión del $6 \%$ y un intervalo de confianza del $95 \%$, el tamaño de muestra era de 260 . Se le aumentó un 10 \% por problemas en la recolección de datos, debiendo revisar 286 indicaciones de transfusión. El muestreo fue estratificado proporcional al tamaño del estrato según el informe estadístico de los hemocomponentes transfundidos el año 2001. El método de selección dentro de cada estrato fue el aleatorio simple. El estudio fue aprobado por el comité de ética hospitalario.

\section{INDICACIÓN DE TRANSFUSIÓN SANGUIINEA}

Consideramos como evento de transfusión de un hemocomponente si el paciente recibía glóbulos rojos (GR), plasma fresco congelado (PFC), concentrado plaquetario o crioprecipitado, en un período de 24 horas (de medianoche a medianoche siguiente). Solo se consideró una indicación por tipo de hemocomponente. Esto se basó en la premisa de que el hemocomponente transfundido durante el período de 24 horas constituye un evento de transfusión que tendría el mismo criterio de indicación ${ }^{(10)}$.

\section{GUÍA DE TRANSFUSIÓN DE COMPONENTES DE LA SANGRE}

Esta guía, elaborada en el Departamento de Patología Clínica del Hospital Rebagliati, señala los criterios de indicación inadecuada para cada hemocomponente. Estos criterios se tomaron de normas y guías internacionales: "Parámetros prácticos para la transfusión de glóbulos rojos del Colegio Americano de Patólogos" (11), "Guías prácticas para la terapia con componentes sanguíneos de la Sociedad Americana de Anestesiología" (3), "Guías para la transfusión de glóbulos rojos y plasma en adultos de la Sociedad Canadiense de Medicina" (12), "Terapia de transfusión de la Asociación Americana de Bancos de Sangre" (13), "Transfusión de plaquetas en pacientes con cáncer de la Sociedad Americana de Oncología Clínica" ${ }^{14)}$ así como otros artículos de revisión ${ }^{(10,15-18)}$. La Tabla 1 resume algunos criterios inadecuados considerados en la guía elaborada.

Para considerar que el perfil de coagulación estaba alterado, debía tener un tiempo de protrombina (TP) o un tiempo de tromboplastina parcial (TTP), antes de la he-

Tabla 1. Indicaciones inadecuadas de transfusión sanguínea según protocolo del Hospital Rebagliati 2002.

\begin{tabular}{ll}
\hline \multicolumn{1}{c}{ Situación } & \multicolumn{1}{c}{\begin{tabular}{c}
\multicolumn{1}{c}{ Criterio inadecuado } \\
de transfusión
\end{tabular}} \\
\hline $\begin{array}{l}\text { Transfusión de } \\
\text { glóbulos rojos en } \\
\text { anemia aguda }\end{array}$ & $\begin{array}{l}\text { Con } \mathrm{Hb}>10 \mathrm{~g} / \mathrm{dL} \\
\text { Como expansor de la volemia }\end{array}$ \\
$\begin{array}{l}\text { Transfusión de } \\
\text { glóbulos rojos } \\
\text { en profilaxis } \\
\text { prequirúrgica }\end{array}$ & $\begin{array}{l}\text { Profilaxis prequirúrgica con } \mathrm{Hb}>10 \\
\mathrm{~g} / \mathrm{dL}\end{array}$ \\
$\begin{array}{l}\text { Expansor de la volemia } \\
\text { Transfusión de } \\
\text { plasma fresco } \\
\text { congelado }\end{array}$ & $\begin{array}{l}\text { Fundip edemas } \\
\text { Únicamente perfil de coagulación } \\
\text { alterado (sin sangrado ni previo a } \\
\text { procedimiento invasivo o quirúrgico) }\end{array}$ \\
$\begin{array}{l}\text { PTI (excepto en hemorragia que } \\
\text { Transfusión de } \\
\text { plaquetas en } \\
\text { escenario clínico y } \\
\text { oncohematológico }\end{array}$ & $\begin{array}{l}\text { pone en peligro la vida) } \\
\text { PTT y trombocitopenia inducida por } \\
\text { heparina (excepto en hemorragia } \\
\text { que pone en peligro la vida) } \\
\text { Disfunción plaquetaria debido a } \\
\text { uremia. }\end{array}$ \\
\hline
\end{tabular}

PTI: púrpura trombocitopénica idiopática; PTT: púrpura trombótica trombocitopénica 
motransfusión, de 1,5 a más veces el valor del control normal. Los valores de control del laboratorio del hospital para el TP y TTP fueron $12 \pm 2$ segundos y $38 \pm 6$ segundos respectivamente.

\section{VALORACIÓN DE LA INDICACIÓN DE TRANSFUSIÓN}

Se revisaron las historias clínicas de los pacientes transfundidos con algún hemocomponente para ver su estado clínico y sus resultados de exámenes de laboratorio. Luego de contrastar esta información con la Guía de transfusión elaborada se procedió a valorar el criterio clínico aplicado como adecuado o inadecuado.

\section{ANÁLISIS ESTADÍSTICO}

Los datos se ingresaron al programa Epilnfo (versión 6.04; CDC, Atlanta, GA). Se aplicó estadística descriptiva, mostrando los datos con frecuencias y porcentajes; obteniendo las prevalencias de transfusión inadecuada según servicio de hospitalización y tipo de hemocomponente.

\section{RESULTADOS}

Se evaluaron 311 indicaciones de transfusiones de hemocomponentes (917 unidades para 241 pacientes) con lo que se cubrió el tamaño de muestra requerido. Se incluyeron 132 indicaciones de GR, 100 de PFC, 54 de plaquetas, y 25 de crioprecipitado. Se encontró prescripciones inadecuadas en 121 de ellas (38,9\%).

Los GR se usaron inadecuadamente en $25 \%$ (33/132), el PFC en $59 \%$ (59/100), las plaquetas en $13 \%(7 / 54)$ y el crioprecipitado en $88 \%(22 / 25)$. En el servicio de medicina, el 36,8 \% (32/87) de las indicaciones de transfusión fueron inadecuadas, en cirugía el $50,5 \%$ (46/91), en las $\mathrm{UCl}$ el $33,7 \%(29 / 86)$ y en emergencia el 29,8\% (14/47). En la Tabla 2 se aprecia el porcentaje de prescripción inadecuada según servicio $y$ hemocomponente.

\section{CRITERIOS INADECUADOS USADOS EN LA INDICACIÓN DE GR}

La administración de GR bajo la indicación de hemorragia perioperatoria $<25 \%$ de la volemia fue la prescripción con mayor frecuencia de mal uso, todos ellos en sala de operaciones (diez casos). Asimismo, se encontró siete indicaciones inadecuadas de administración de GR en anemia aguda con $\mathrm{Hb}>10 \mathrm{~g} / \mathrm{dL}$ (tres en Cir-SOP, dos en $\mathrm{UCl}$, uno en Med y uno en Cir-Hosp) y siete por anemia aguda o hemorragia perioperatoria con $\mathrm{Hb}$ entre 6 y $10 \mathrm{~g} / \mathrm{dL}$ sin factores de riesgo de hipoxia tisular (tres en Med, tres en Emg y uno en Cir-Hosp).

Con relación a casos de anemia crónica, se encontraron cuatro indicaciones inadecuadas de transfusión en pacientes con $\mathrm{Hb}$ entre 5 y $8 \mathrm{~g} / \mathrm{dL}$ sin síntomas de hipoxia (servicio de Medicina y Emergencia, dos casos cada uno), y una indicación en un paciente con $\mathrm{Hb}>8$ g/dL (Cir-Hosp).

También se halló cuatro prescripciones inadecuadas en casos de profilaxis prequirúrgica con $\mathrm{Hb}$ entre $8 \mathrm{y}$ $10 \mathrm{~g} / \mathrm{dL}$, no justificadas ni por el tipo de cirugía ni por factores asociados a hipoxia tisular (dos en Med, uno en UCl, uno en Cir-Hosp).

\section{CRITERIOS INADECUADOS USADOS EN LA INDICACIÓN DE PFC}

El criterio inadecuado utilizado con mayor frecuencia fue el sangrado leve sin perfil alterado (nueve en Cir-SOP, dos en Emg y dos en $\mathrm{UCl}$ ) seguido de hipoalbuminemia mas edema (seis en Med, cuatro en Cir-Hosp) y perfil alterado sin sangrado ni cirugía próxima (tres en Emg, dos en Med, dos en UCl y dos en Cir-Hosp). También se encontró ocho prescripciones inadecuadas por la indicación de transfusión en prequirúrgico sin perfil alterado (tres en Emg, dos en Med, dos en CirHosp y uno en $\mathrm{UCl}$ ) y ocho por uso en pacientes que presentaban solamente edemas (seis en Med, uno en $\mathrm{UCl}$, uno en Cir-Hosp).

Tabla 2. Valoración de la indicación de transfusión según servicio y hemocomponente.

\begin{tabular}{|c|c|c|c|c|c|c|c|c|}
\hline \multirow{3}{*}{ Servicio } & \multicolumn{2}{|c|}{ Glóbulos rojos } & \multicolumn{2}{|c|}{ Plasma fresco congelado } & \multicolumn{2}{|c|}{ Plaquetas } & \multicolumn{2}{|c|}{ Crioprecipitado } \\
\hline & Adecuada & Inadecuada & Adecuada & Inadecuada & Adecuada & Inadecuada & Adecuada & Inadecuada \\
\hline & N (\%) & $\mathbf{N}(\%)$ & N (\%) & $\mathbf{N}(\%)$ & $\mathbf{N}(\%)$ & N (\%) & $\mathbf{N}(\%)$ & $\mathbf{N}(\%)$ \\
\hline Medicina & $24(75)$ & $8(25)$ & $7(27)$ & $19(73)$ & $24(86)$ & $4(14)$ & -- & $1(100)$ \\
\hline Cirugía & $34(67)$ & $17(33)$ & $8(25)$ & $24(75)$ & $3(60)$ & $2(40)$ & -- & $3(100)$ \\
\hline $\mathrm{UCl}$ & $22(88)$ & $3(12)$ & $21(72)$ & $8(28)$ & $11(92)$ & $1(8)$ & $3(15)$ & $17(85)$ \\
\hline Emergencia & $19(79)$ & $5(21)$ & $5(38)$ & $8(62)$ & $9(100)$ & -- & -- & $1(100)$ \\
\hline
\end{tabular}

$\mathrm{N}(\%)$, número de indicaciones de transfusión. 
Otras indicaciones inadecuadas fueron: postquirúrgico sin perfil alterado (cinco en Cir-Hosp, uno en UCl), hipoalbuminemia solamente (dos en Med), hepatopatía grave más perfil alterado sin sangrado previo a procedimiento invasivo (uno en Med), transfusión masiva < 1,5 volemias de recambio (uno en Cir-SOP) y expansor de la volemia (uno en $\mathrm{UCl}$ ). Fue constante observar, en todos los servicios, que una leve prolongación de los tiempos de coagulación sin llegar al umbral conocido fue considerada como "perfil alterado".

\section{CRITERIOS INADECUADOS USADOS EN LA INDICACIÓN DE PLAQUETAS}

Las plaquetas fueron el hemocomponente mejor utilizado. Las especialidades de oncología y hematología son las que más usaron los concentrados plaquetarios. Se encontró dos prescripciones inadecuadas por trombocitopenia crónica, severa y estable sin síntomas y sin tratamiento activo en el Servicio de Medicina.

Otras indicaciones inadecuadas fueron: paciente con sepsis y recuento plaquetario $>43 \mathrm{000} / \mu \mathrm{l}$ (uno en $\mathrm{UCl}$ ), procedimiento invasivo mayor con recuento plaquetario $>50$ 000/ $\mu$ l (un caso en Medicina por colocación de un catéter central), hemorragia activa con recuento plaque-

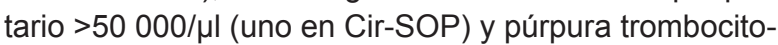
pénica idiopática (uno en Med).

\section{CRITERIOS USADOS EN LA INDICACIÓN DE CRIOPRECIPITADO}

El crioprecipitado se usó fundamentalmente en las $\mathrm{UCl}$, en el escenario postoperatorio de cirugías mayores; a estos pacientes se les transfundió incorrectamente crioprecipitado debido a un "perfil alterado", incluso solo debido a una leve prolongación del TP o TTP o también en forma empírica bajo la suposición de una deficiencia de factores de coagulación y de fibrinógeno debido a la cirugía. Las indicaciones inadecuadas más frecuentes se resumen en la Tabla 3.

Tabla 3. Transfusión de hemocomponentes. Indicaciones inadecuadas más frecuentes (Hospital Rebagliati 2002).

\begin{tabular}{|c|c|}
\hline Indicación según tipo & $\mathrm{N}(\%)$ \\
\hline \multicolumn{2}{|l|}{ Glóbulos Rojos ( $n=132)$} \\
\hline $\begin{array}{l}\text { Hemorragia perioperatoria }<25 \% \\
\text { de la volemia }\end{array}$ & $10(7,6)$ \\
\hline \multicolumn{2}{|l|}{ Plasma fresco congelado $(n=100)$} \\
\hline Sangrado leve sin perfil alterado & $13(13,0)$ \\
\hline Hipoalbuminemia + edema & $10(10,0)$ \\
\hline Perfil alterado sin sangrado y sin cirugía próxima & $9 \quad(9,0)$ \\
\hline \multicolumn{2}{|c|}{ Crioprecipitado $(n=25)$} \\
\hline Postquirúrgico + perfil normal + fibrinogenemia & $9(36,0)$ \\
\hline
\end{tabular}

\section{DISCUSIÓN}

La alta proporción $(38,9 \%)$ de indicación inadecuada de transfusión que se encontró en el Hospital Rebagliati guarda similitud con otras publicaciones. Así, en 14 Hospitales de Australia (19), se encontró transfusiones inapropiadas de $33 \%$ para las plaquetas, $37 \%$ para el PFC y $62 \%$ para el crioprecipitado. En un hospital general de Venezuela (20) la prevalencia de uso inapropiado de productos sanguíneos fue de $48,7 \%$; según servicios, el uso inapropiado fue: $28 \%$ medicina, $64 \%$ cirugía, $44 \%$ emergencia y $53 \%$ obstetricia.

Hebert et al. (17) encontraron que la tasa de mortalidad global fue significativamente más baja en el grupo de pacientes con estrategia restrictiva de transfusión $(\mathrm{Hb}$ de 7 a $9 \mathrm{~g} / \mathrm{dL}$ ) en comparación con una liberal ( $\mathrm{Hb}$ de 10 a $12 \mathrm{~g} / \mathrm{dL}$ ). Este fue el primer estudio que sugirió que no hay beneficio en mantener un nivel de hemoglobina mayor de $10 \mathrm{~g} / \mathrm{dL}$ en pacientes críticamente enfermos. French et al. (21) afirman que la publicación de estos resultados mejoró la práctica transfusional en las $\mathrm{UCl}$ de Australia donde la tasa de transfusión inapropiada de GR fue de solo el $3 \%$.

Goodnough et al. (22) señalan que un nivel de $\mathrm{Hb}$ de 8 $\mathrm{g} / \mathrm{dL}$ parece ser el umbral apropiado para la transfusión en pacientes quirúrgicos sin factores de riesgo para isquemia, mientras que el umbral de $10 \mathrm{~g} / \mathrm{dL}$ podría estar justificado para pacientes considerados en alto riesgo. Cortina y López de Roux ${ }^{(16)}$ señalan que la práctica de la anestesia quirúrgica ha sido guiada por el supuesto que un valor de $\mathrm{Hb}<$ de $10 \mathrm{~g} / \mathrm{dL}$ es una indicación para transfusión. Actualmente, se señala que en lugar de corregir un valor de laboratorio, deberán tomarse en consideración múltiples factores como la duración de la anemia, extensión de la cirugía, probabilidad de una pérdida masiva de sangre, así como otras condiciones coexistentes (deterioro de la función respiratoria, gasto cardiaco inadecuado, isquemia miocárdica o cerebrovascular y enfermedad vascular periférica). Si una persona está sana y no existen factores de riesgo adicionales asociados a la naturaleza de la cirugía, los niveles de $\mathrm{Hb}$ iguales a $8 \mathrm{~g} / \mathrm{dL}$, o incluso menores, serán bien tolerados si el paciente está bien controlado ${ }^{(16)}$. En el caso de los pacientes testigos de Jehová que se someten a una cirugía mayor se evidenció que con un nivel de $\mathrm{Hb}$ tan bajo como 7 u $8 \mathrm{~g} / \mathrm{dL}$ son fisiológicamente bien tolerados ${ }^{(23,24)}$.

Los criterios de profilaxis prequirúrgica con puntos de corte de 8 y $10 \mathrm{~g} / \mathrm{dL}$ de $\mathrm{Hb}$ no están señalados en ninguno de los consensos referidos. En la presente investigación se consideran estos umbrales como una propuesta ${ }^{(15,23-25)}$. 
En sala de operaciones se vio la mayor cantidad de prescripciones inadecuadas de GR, todas ellas por hemorragias perioperatorias < $25 \%$ de la volemia. Se observó que hemorragias entre 300 y $700 \mathrm{~mL}$ fueron condicionantes para iniciar la transfusión. Asimismo, se identificaron casos de transfusión conjunta de GR y PFC con sangrados de 300 a $500 \mathrm{~mL}$. En los pacientes que se someterán a cirugía es esencial que se les ofrezca un tratamiento alternativo al uso de sangre alogénica como son la hemodilución intraoperatoria y el almacenaje preoperatorio de sangre autóloga. En el hospital son muy escasos los pacientes que se benefician con estas terapias más seguras.

El plasma fresco congelado fue el producto sanguíneo peor usado en la práctica transfusional del Hospital Rebagliati. Es necesario conocer la definición correcta de perfil de coagulación alterado (TTP o TP es > a 1,5 veces el valor de control normal). Además de presentar un perfil alterado, es necesario otra condición adicional.

En las salas de operaciones, el uso innecesario de PFC se dio en sangrados leves que oscilaban entre 200 y $800 \mathrm{~mL}$. Como se sabe, en condiciones de resucitación isovolémica es necesario una pérdida 1,5 volemias (alrededor de 6 a 7 litros de sangrado) para que recién se desarrolle una coagulopatía dilucional. Cabe tener en cuenta también que una de las causas de la prolongación del TTP es la colección incorrecta de la muestra sanguínea debida, sobre todo, a una venipuntura traumática ${ }^{(26)}$.

En las UCl a los pacientes en su periodo posquirúrgico inmediato de cirugía mayor se les indicó PFC sin los resultados laboratoriales del TP o TTP, es decir, la indicación fue empírica. El consenso señala que si el TP o TTP no pueden ser obtenidos en el tiempo requerido, los pacientes pueden beneficiarse de la transfusión empírica con PFC. Aunque un análisis detenido del historial de perfiles de coagulación anteriores a la cirugía, el volumen de sangrado operatorio y del contexto clínico global del paciente, nos indicaba claramente que se pudo racionalizar un tanto su uso. Estos mismos pacientes en su postquirúrgico inmediato, muchas veces recibieron en forma conjunta GR, PFC, plaquetas y crioprecipitado (transfusiones empíricas), mayormente dos o tres de estos componentes, teniendo como objetivo el mantener una hemostasia adecuada en el paciente. Conviene decir que el uso de los componentes debe ser analizado independientemente unos de otros. En la mayor parte de estas indicaciones conjuntas no todos los hemocomponentes usados estaban justificados. Se debe considerar que una gran proporción de las indicaciones inadecuadas evaluadas en este estudio se debió a que los médicos exageraron al tomar precauciones.
En los países desarrollados, el interés en los procesos de mejoramiento para guiar la decisión de transfusión se ha incrementado en la últimas dos décadas, no solo porque una transfusión inapropiada conlleva riesgos médicos, sino también debido a que una transfusión inapropiada es costosa. ${ }^{(2)}$ La elevada frecuencia de prescripción inadecuada de hemocomponentes en el Hospital Rebagliati crea la necesidad de reducirlas empleando estrategias como programas de educación continua, publicación de guías prácticas, presentación de casos y auditorias médicas. La identificación de los criterios específicos de indicación inapropiada orienta a diseñar un mejor programa educativo hacia el personal médico.

García et al.(27) en un hospital de España demostraron que el conocimiento de las guías de transfusión por parte del personal médico mejora el uso de componentes sanguíneos; así, observaron un incremento significativo de órdenes de transfusión apropiadas de 74 a $87 \%$. En otro estudio Tuckfield et al. (28) demuestran que con una monitorización prospectiva de solicitudes de hemocomponentes se puede reducir las tasas de transfusión inadecuada.

Como limitaciones del estudio, mencionaremos que la presente investigación se realizó en un hospital de la seguridad social en un corto periodo y se consideró solamente cuatro servicios de hospitalización, por lo que los resultados no se pueden extrapolar a otras instituciones. Asimismo, al ser un trabajo descriptivo en base a historias clínicas, no se puede tener la certeza que la información registrada haya sido la indicada. Tampoco se obtuvieron igual número de indicaciones para los cuatro hemocomponentes evaluados, especialmente con el crioprecipitado, del cual se tuvo 25 indicaciones, por lo que las comparaciones no deben ser literales. A pesar de publicaciones más recientes $(2004$ - 2011) sobre el tema de transfusión de hemocomponentes, los lineamientos prácticos de la guía utilizada en la presente investigación permanecen vigentes.

En conclusión, existe una considerable proporción de indicación inadecuada de transfusión sanguínea en el Hospital Rebagliati. Entre las causas más importantes de este sobreuso de hemocomponentes están el desconocimiento de los lineamientos de transfusión y también debido a que los clínicos exageran en tomar precauciones. La identificación de los criterios clínicos específicos de indicación inapropiada orienta a diseñar una mejor estrategia educativa hacia un uso más racional de la sangre.

\section{Fuentes de financiamiento}

Autofinanciado. 


\section{Conflictos de interés}

El autor declara no tener conflictos de interés en la publicación de este artículo.

\section{REFERENCIAS BIBLIOGRÁFICAS}

1. Corwin HL. Blood transfusion: first, do no harm! Chest 1999;116(5):1149-50.

2. Isbister JP. Risk Management in Transfusion Medicine. Transfusion Medicine Reviews. 1996;10(3):183-202.

3. Practice Guidelines for blood component therapy: A report by the American Society of Anesthesiologists Task Force on Blood Component Therapy. Anesthesiology. 1996;84(3):732-47.

4. Mintz D. Transfusion therapy: clinical principles and practice. $1^{\text {st }}$. Ed. Bethesda, MD: American Association of Blood Banks; 1999.

5. Toy P. Guiding the Decision to Transfuse. Arch Pathol Lab Med. 1999;123(7):592-4.

6. Metz J, McGrath KM, Copperchini ML, Haeusler M, Haysom HE, Gibson PR, et al. Appropriateness of transfusions of red cells, platelets and fresh frozen plasma. An audit in a tertiary care teaching Hospital. Med J Aust. 1995;162(11):572-7.

7. Ghali WA, Palepu A, Paterson WG. Evaluation of red blood cell transfusion practices with the use of preset criteria. CMAJ. 1994;150(9):1449-54.

8. Luk C, Eckert KM, Barr RM, Chin-Yee IH. Prospective audit of the use of fresh-frozen plasma, based on Canadian Medical Association transfusion guidelines. CMAJ. 2002;166(12):1539-40.

9. Beloeil H, Brosseau M, Benhamou D. Transfusion of fresh frozen plasma (FFP): audit of prescriptions. Ann Fr Anesth Reanim. 2001;20(8):686-92.

10. Corwin HL, Parsonnet KC, Gettinger A. RBC transfusion in the ICU. Is there a reason? Chest. 1995;108(3):767-71.

11. Simon TL, Alverson DC, AuBuchon J, Cooper ES, DeChristopher PJ, Glenn GC, et al. Practice parameter for the use of red blood cell transfusions: developed by the Red Blood Cell Administration Practice Guideline Development Task Force of the College of American Pathologists. Arch Pathol Lab Med. 1998;122(2):130-8.

12. Gatien G. Guideling for red blood cell and plasma transfusion for adults and children. CMAJ. 1997;156(11):S1-S24.

13. American Association of Blood Banks. Technical Manual. Glenbrook Road $13^{\circ} \mathrm{ed}$. Bethesda: Maryland: American Association of Blood Banks; 1999.

14. Schiffer CA, Anderson KC, Bennett CL, Bernstein S, Elting LS, Goldsmith M, et al. Platelet Transfusion for Patients with Cancer: Clinical Practice Guidelines of the American Society of Clinical Oncology. J Clin Oncol. 2001;19(5):1519-38.

15. Cortés-Buelvas A, Rovetto P, Percira F, Gómez A, Granados M. Medicina transfusional: componentes sanguíneos usuales y especiales. Bogotá: Asociación Colombiana de Facultades de Medicina; 2008.
16. Cortina-Rosales L, López de Roux M. Utilización de la Sangre y sus componentes celulares. Rev Cubana Hematol Inmunol Hemoter. 2000;16(2):78-89.

17. Hébert PC, Wells G, Blajchman MA, Marshall J, Martin C, Pagliarello G, et al. A multicenter, randomized, controlled clinical trial of transfusion requirements in critical care. Transfusion Requirements in Critical Care Investigators, Canadian Critical Care Trials Group. N Engl J Med. 1999;340(6):409-17. Erratum in: N Engl J Med 1999; 340(13):1056.

18. Wandt H, Ehninger G, Gallmeier WM. New strategies for prophylactic platelet transfusion in patients with hematologic diseases. Oncologist. 2001;6(5):446-50.

19. Schofield WN, Rubin GL, Dean MG. Appropriateness of platelet, fresh plasma and cryoprecipitate transfusion in New South Wales public Hospitals. MJA. 2003;178:117-21.

20. Marti-Carvajal AJ, Munoz-Navarro SR, Pena-Marti GE, Comunian G. An audit of appropriate use of blood products in adult patients in a Venezuelan general university Hospital. Int J Qual Health Care. 1999;11(5):391-5.

21. French CJ, Bellomo R, Finfer SR, Lipman J, Chapman M, Boyce NW. Appropriateness of red blood cell transfusion in Australasian intensive care practice. Med J Aust. 2002;177(10):548-51.

22. Goodnough L, Bracher M, Kanter M, Aubuchon J. Transfusion Medicine: Blood Transfusion. N Engl J Med. 1999;340:438-47.

23. Spence RK, Carson JA, Poses R, McCoy S, Pello M, Alexander $\mathrm{J}$, et al. Elective surgery without transfusion: influence of preoperative hemoglobin level and blood loss on mortality. Am J Surg. 1990;159(3):320-4.

24. Kitchen CS. Are transfusions overrated? Surgical outcome of Jehovah's Witnesses. Am J Med. 1993;94(2):117-9.

25. Carson JL, Duff A, Berlin JA, Lawrence VA, Poses RM, Huber EC, et al. Perioperative blood transfusion and postoperative mortality. JAMA. 1998;279(3):199-205.

26. Triplett DA. Coagulation and bleeding disorders: Review and update. Clinical Chemistry. 2000;46(8 Pt 2):1260-9.

27. García Gala JM, Rodríguez Vicente P, Bernal del CastiIlo T, Martínez Revuelta E, Corte Buelga JR, Rodríguez Luengo J, et al. Adequacy of blood component transfusion according to previously established criteria. Sangre (Barc). 1996;41(1):19-23.

28. Tuckfield A, Haeusler MN, Grigg AP, Metz J. Reduction of inappropriate use of blood products by prospective monitoring of transfusion request forms. Med J Aust. 1997;167(9):473-6.
Correspondencia: Wilfredo Flores Paredes

Dirección: Av. Brasil 1320 Dpto. F, Lima 13, Perú.

Teléfono: (511) 3242983 anexo 4161

Correo electrónico: wido2@hotmail.com 